\title{
Open
}

\section{Impaired innate interferon induction in severe therapy resistant atopic asthmatic children}

\author{
MR Edwards ${ }^{1,2,3,6}$, N Regamey ${ }^{4,6}$, M Vareille ${ }^{4}$, E Kieninger ${ }^{4}$, A Gupta $^{2,5}$, A Shoemark ${ }^{2,5}$, S Saglani ${ }^{2,5}$,

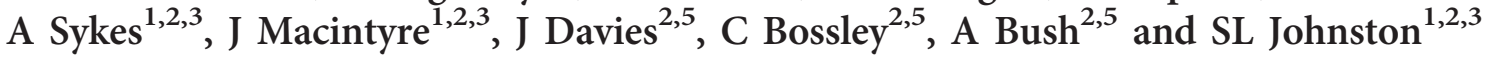

Deficient type I interferon- $\beta$ and type III interferon- $\lambda$ induction by rhinoviruses has previously been reported in mild/ moderate atopic asthmatic adults. No studies have yet investigated if this occurs in severe therapy resistant asthma (STRA). Here, we show that compared with non-allergic healthy control children, bronchial epithelial cells cultured ex vivo from severe therapy resistant atopic asthmatic children have profoundly impaired interferon- $\beta$ and interferon- $\lambda$ mRNA and protein in response to rhinovirus (RV) and polylC stimulation. Severe treatment resistant asthmatics also exhibited increased virus load, which negatively correlated with interferon mRNA levels. Furthermore, uninfected cells from severe therapy resistant asthmatic children showed lower levels of Toll-like receptor-3 mRNA and reduced retinoic acid inducible gene and melanoma differentiation-associated gene 5 mRNA after RV stimulation. These data expand on the original work, suggesting that the innate anti-viral response to RVs is impaired in asthmatic tissues and demonstrate that this is a feature of STRA.

\section{INTRODUCTION}

Acute viral infections are the most frequent cause of asthma exacerbations, and are therefore responsible for the majority of the morbidity, mortality, and healthcare costs attributed to asthma. ${ }^{1-4}$ Despite treatment with oral or inhaled corticosteroids (ICS), $\beta_{2}$ agonists and their combinations; asthma exacerbations continue to occur and represent a major unmet need in medical practice. ${ }^{5-8}$ Children with severe therapy resistant asthma (STRA), have asthma-related symptoms, continued morbidity, hospitalizations, and asthma exacerbations despite high-dose therapy. ${ }^{9}$ Atopic STRA children previously exhibited variable eosinophillic airway inflammation yet submucosal mast cell, neutrophil, and lymphocyte counts did not significantly differ from non-asthmatic control groups. Increased bronchoalveolar lavage (BAL) Th2 cytokines are not a defining feature of STRA, despite the presence of atopy. ${ }^{10}$ More research is needed to understand potential mechanisms of asthma exacerbations in severe asthma and the identification of new therapeutic targets represent a major research goal.
Recently, innate anti-viral responses to rhinovirus (RV) infection have been shown to be impaired in cells from asthmatics. ${ }^{11-15}$ Upon infection with RV, bronchial epithelial cells (BECs) respond by producing innate interferon (IFN) production composed of type I IFN- $\beta$, and the type III IFN$\lambda s ;{ }^{16-18}$ IFN- $\lambda s$ are a newly identified anti-viral cytokine family, which use a unique receptor complex ${ }^{19}$ yet have similar properties to the type I IFNs IFN- $\beta$ and IFN- $\alpha .{ }^{20}$ IFN $-\beta$, rather than the numerous IFN- $\alpha$ s are the most common type I IFN produced by BECs, ${ }^{21}$ and both IFN- $\beta$ and IFN- $\lambda$ s signal via their respective receptor complexes to induce a well-ordered program of transcription, resulting in the upregulation of IFNstimulated genes (ISGs). ${ }^{22,23}$ The ISGs elicit the anti-viral response, through degrading virus RNA, preventing virusassociated protein trafficking and/or virion assembly, and inducing apoptosis. IFN from infected cells act in a positive feedback loop and prime adjacent, uninfected cells for rapid induction of anti-viral immunity through upregulation of more IFN, innate receptors, and ISGs. ${ }^{24,25}$ BECs cultured from bronchial brushings of mild-moderate atopic asthmatic adults

\footnotetext{
${ }^{1}$ Respiratory Medicine, St Mary's Campus, National Heart Lung Institute, London, UK. ${ }^{2}$ MRC and Asthma UK Centre for Allergic Mechanisms of Asthma, London, UK. ${ }^{3}$ Centre for Respiratory Infections, Imperial College, London, UK. ${ }^{4}$ Division of Paediatric Respiratory Medicine, Department of Paediatrics and Department of Clinical Research, Inselspital, Bern University Hospital, and University of Bern, Bern, Switzerland. ${ }^{5}$ Respiratory Paediatrics, Royal Brompton NHS Trust, Imperial College, London, UK and ${ }^{6}$ These authors contributed equally to this work. Correspondence: MR Edwards (michael.edwards@imperial.ac.uk)
} 
have demonstrated deficient IFN- $\beta$ mRNA and protein upon RV16 infection ex vivo, decreased RV16 induced apoptosis, and increased virus replication compared with control cells from non-atopic non-asthmatics (NANA). ${ }^{12}$ The asthmatic BECs had similar mRNA and protein of the pro-inflammatory cytokines IL-6 and RANTES compared with non-asthmatics, suggesting that this deficiency was associated specifically with IFN production. The deficient IFN- $\beta$ observed was not related to GC use, as GC treated and GC naive asthmatic individuals exhibited similar levels of reduced RV induced IFN- $\beta$. In a related study, ${ }^{13}$ the type III IFN- $\lambda$ family was studied in the BECs from adults sampled by Wark et al. The BECs from asthmatic subjects also showed deficient IFN- $\lambda 1$ and IFN- $\lambda 2 / 3$ mRNA and protein compared with controls, which negatively correlated with RV16 replication. Furthermore, the importance of IFN- $\lambda$ was demonstrated in vivo using an experimental RV challenge model in asthmatics. ${ }^{13}$ BAL cells from mildmoderate atopic asthmatic adult donors stimulated with RV16 ex vivo demonstrated deficient levels of IFN- $\lambda 1$ and IFN- $\lambda 2 / 3$ protein and mRNA compared with NANA controls. The abundance of ex vivo derived IFN- $\lambda 1$ and IFN $-\lambda 2 / 3$ protein levels negatively correlated with RV load, cold score, reductions in lung function and markers of airway inflammation after experimental challenge in vivo. ${ }^{13}$ These data demonstrate IFN$\lambda s$ to be associated with severity of asthma exacerbations and also highlight that adult asthmatics are deficient in this important anti-viral cytokine family.

While the initial findings concerning IFN $-\beta$ and IFN $-\lambda$ impairment have been reproduced in a study using asthmatic BECs cultured with polyIC; ${ }^{14}$ two recent studies have failed to observe impaired IFN production in BECs cultured from asthmatics, suggesting that impaired IFN may not be so easily observable or is related to a subset of asthmatics. ${ }^{22,26}$ Furthermore, impaired IFN expression in BAL macrophages was recently confirmed by a study of mild-moderate asthmatic adults, ${ }^{27}$ and impaired IFN upon infection has also been observed with other respiratory viruses including Newcastle disease virus and respiratory syncytial virus infection of peripheral blood mononuclear cells ${ }^{28,29}$ and influenza infection of purified plasmacytoid dendritic cells (pDCs). ${ }^{30}$ Together, the data suggest that impaired IFN responses in asthma may not be restricted to BECs and are not limited to RV infection.

In the present report, we have investigated IFN expression upon RV infection and stimulation with the Toll-like receptor-3 (TLR3) ligand polyIC in ex vivo cultured BECs. We observed that IFN- $\beta$, IFN- $\lambda 1$, and IFN- $\lambda 2 / 3$ mRNA levels are up to 600 fold lower in BECs from STRA children compared with NANA controls. Basal TLR3 levels were also lower in STRA BECs, and at $24 \mathrm{~h}$ post-infection, the virus induced RIG-like helicases (RLHs) retinoic acid inducible gene (RIG-I) and melanoma differentiation-associated gene 5 (MDA5) mRNA were also diminished in STRA BECs. STRA children also showed elevated RV replication yet normal CXCL8/IL-8 and CXCL5/ENA-78 pro-inflammatory cytokine mRNA levels. RV induced IFN $-\beta$, IFN $-\lambda 1$, and IFN- $\lambda 2 / 3$ mRNA levels were not associated with age, IgE levels, allergen reactivity, BAL and sputum neutrophils and eosinophils, or lung function. Thus, deficient innate IFN responses are present in BECs from severe therapy resistant asthmatic children.

\section{RESULTS}

STRA BECs exhibited impaired IFN- $\beta$ and IFN- $\lambda 2 / 3$ mRNA abundance at 8 and $24 \mathrm{~h}$ following $\mathrm{RV}$ infection.

We first assessed the expression of IFN- $\beta$, IFN- $\lambda 1$, IFN- $\lambda 2 / 3$ mRNA following RV16 and RV1B infection at $8 \mathrm{~h}$ in both STRA and NANA using real-time quantitative PCR, presenting the data as normalized copy number. At $8 \mathrm{~h}$ post-infection, we observed significant induction of IFN- $\beta$ in NANA $(P<0.05)$ and in STRA $(P<0.01)$ by RV1B but not RV16 when compared with medium. There was a significantly greater IFN- $\beta$ mRNA abundance in NANA compared with STRA in RV16 infected cells $(P<0.05$, fold difference in median, 320$)$ with a similar trend for RV1B-induced IFN- $\beta$ (fold difference in median 5.7), though this trend was not statistically significant (Figure 1a). For IFN- $\lambda 1$, we observed a significant induction by both RV16 and RV1B for NANA $(P<0.05$ for both viruses) and STRA $(P<0.05$ RV16, $P<0.001 \mathrm{RV} 1 \mathrm{~B})$. There was no statistically significant difference in IFN- $\lambda 1$ mRNA abundance between NANA and STRA; however, STRA had lower median levels for both RV16 and RV1B (median fold difference 614 for RV16; 7.4 for RV1B) (Figure 1b). For IFN- $\lambda 2 / 3$ mRNA, we observed a
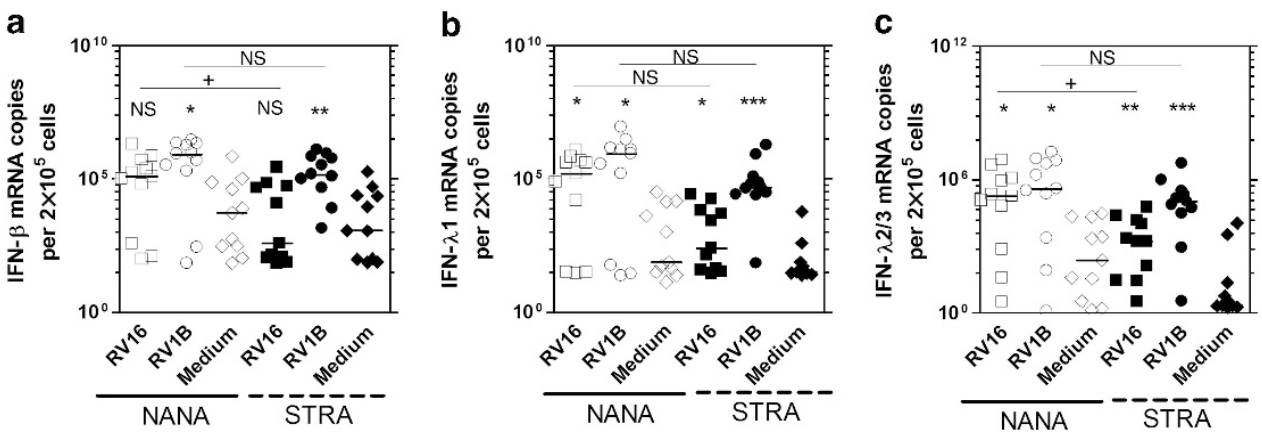

Figure 1 Bronchial epithelial cells (BECs) from severe therapy resistant asthma (STRA) have impaired RV16 induced interferon (IFN)- $\beta$ and IFN- $\lambda 2 / 3$ mRNA expression compared with non-atopic non-asthmatic (NANA) at $8 \mathrm{~h}$. NANA $(n=11)$ and STRA $(n=11)$ BEC cultures were infected with RV16, RV1B, or medium and (a) IFN- $\beta$, (b) IFN- $\lambda 1$, (c) IFN- $\lambda 2 / 3$ mRNA measured at $8 \mathrm{~h}$ post-infection. Horizontal line indicates the median. ${ }^{\star} P<0.05,{ }^{* \star} P<0.01,{ }^{* \star *} P<0.001$ vs. medium ${ }^{+} P<0.05$ as indicated, NS $=$ not significant. 
significant induction by both RV16 and RV1B for NANA $(P<0.05$ for both viruses $)$ and STRA $(P<0.01 \mathrm{RV} 16, P<0.001$ RV1B). STRA had less IFN- $\lambda 2 / 3$ mRNA than NANA for both RV16 and RV1B infected cultures (median fold difference, 109 and 3.8, respectively) although differences were significant for RV16 only $(P<0.05)$ (Figure 1c).

At $24 \mathrm{~h}$ post-infection, each IFN subtype was significantly induced by both RV1B and RV16 $(P<0.01$ at least) with the exception of IFN- $\beta$ in STRA, which was not significantly induced by RV16 (Figures 2a-c). There was significantly less IFN mRNA abundance in STRA compared with NANA for all IFN subtypes, with median fold differences for IFN- $\beta$ of 282 with RV16 $(P<0.01)$, and 23 for RV1B $(P<0.01)$ (Figure 2a), for IFN- $\lambda 1,261$ with RV16 $(P<0.05)$ and 46 for RV1B $(P<0.05)$ (Figure $2 \mathbf{b})$, for IFN- $\lambda 2 / 3$ a fold difference of 131 for RV16 $(P<0.01)$ and 11 for RV1B $(P<0.05)$ was observed (Figure 2c). IFN abundance at $24 \mathrm{~h}$ did not correlate with age (Supplementary Figure S2 online) and was not significantly different between males and females (Supplementary Figure S3 online). We also measured the induction of two non-IFN genes, CXCL8 and CXCL5 and compared expression levels in NANA and STRA to determine whether deficiencies were restricted to IFN induction. We observed that CXCL8 and CXCL5 mRNA abundance was not significantly induced by RV16 or RV1B vs. medium treated controls at either time point and no differences were observed between NANA and STRA for any treatment (data not shown).

\section{Uninfected STRA BECs exhibited lower TLR3 mRNA levels and impaired RV induced RIG-I and MDA5 mRNA abundance at $\mathbf{2 4} \mathrm{h}$}

Having established that IFN mRNA induction at $24 \mathrm{~h}$ was significantly impaired in STRA vs. controls, we next assessed if mRNA levels of the pattern recognition receptors RIG-I, MDA5, and TLR3 were differentially expressed in STRA and healthy control samples. Previously, studies have shown that TLR3, RIG-I, and MDA5 are required for IFN mRNA induction by $\mathrm{RV}^{17,18}$ with TLR3 acting as a likely initial sensor of dsRNA and RIG-I and MDA5 serving as virus and IFNinducible sensors also required for maximal IFN mRNA induction. ${ }^{18} \mathrm{We}$ initially found that at $8 \mathrm{~h}$ post-infection with RV1B or RV16, RIG-I and MDA5 were not significantly induced and that levels were similar in STRA and controls (Supplementary Figure S4 online). TLR3 mRNA was also not induced at $8 \mathrm{~h}$, but was significantly less abundant in STRA samples vs. controls for all treatments $(P<0.05$ at least) (Supplementary Figure S4 online). At 24 h postinfection, we found that in healthy controls RV16 and RV1B both significantly induced RIG-I $(P<0.001$ and $P<0.01$, respectively) (Figure 3a) and MDA5 (Figure 3b) $(P<0.001$
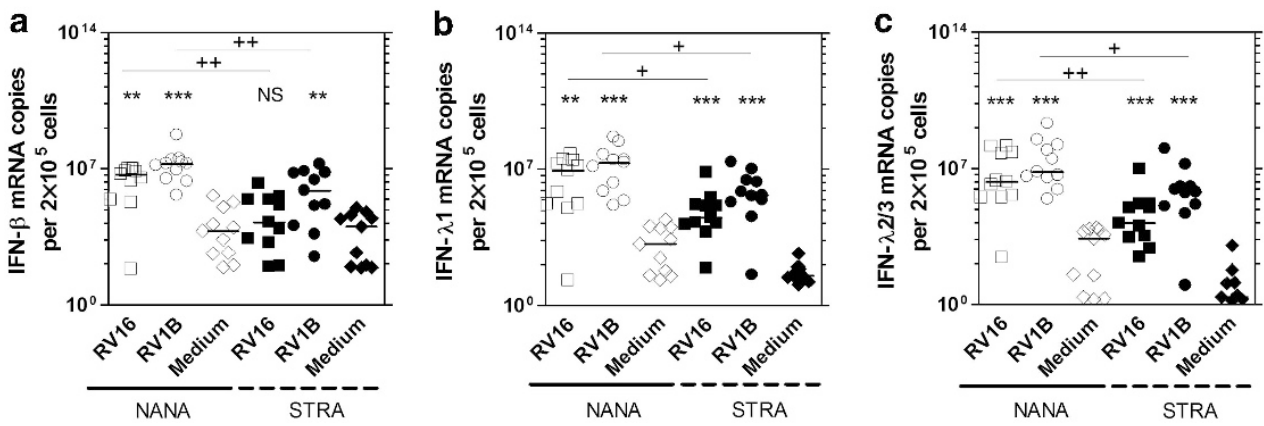

Figure 2 Bronchial epithelial cells (BECs) from severe therapy resistant asthma (STRA) have impaired RV16 and RV1B induced interferon (IFN)- $\beta$, IFN- $\lambda 1$, and IFN- $\lambda 2 / 3$ mRNA compared with non-atopic non-asthmatic (NANA) at $24 \mathrm{~h}$. NANA $(n=11)$ and STRA ( $n=11)$ BEC cultures were infected with RV16, RV1B, or medium and (a) IFN- $\beta$, (b) IFN- $\lambda 1$, (c) IFN- $\lambda 2 / 3$ mRNA measured at $24 \mathrm{~h}$ post-infection. Horizontal line indicates the median ${ }^{\star} P<0.05,{ }^{\star \star} P<0.01,{ }^{* \star} P<0.001$ vs. medium ${ }^{+} P<0.05,{ }^{++} P<0.01$, as indicated, $\mathrm{NS}=$ not significant.
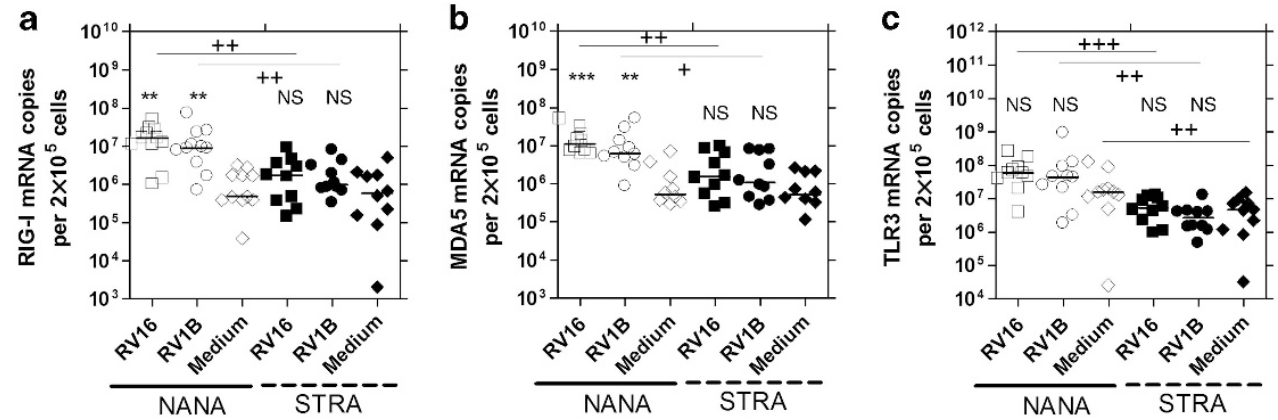

Figure 3 Unstimulated bronchial epithelial cells (BECs) from severe therapy resistant asthma (STRA) have impaired Toll-like receptor-3 (TLR3) mRNA levels and impaired RV16 and RV1B induced RIG-I and MDA5 mRNA compared with non-atopic non-asthmatic (NANA) at 24 h. NANA (RIG-I $n=11$, MDA5 and TLR3 $n=10)$ and STRA $(n=10)$ BEC cultures were infected with RV16, RV1B, or medium and (a) RIG-I, (b) MDA5, (c) TLR3 mRNA measured at $24 \mathrm{~h}$ post-infection. Horizontal line indicates the median ${ }^{*} P<0.05,{ }^{* *} P<0.01,{ }^{* * *} P<0.001$ vs. medium ${ }^{+} P<0.05,{ }^{+}+P<0.01$, $+++P<0.001$ as indicated, NS $=$ not significant. 

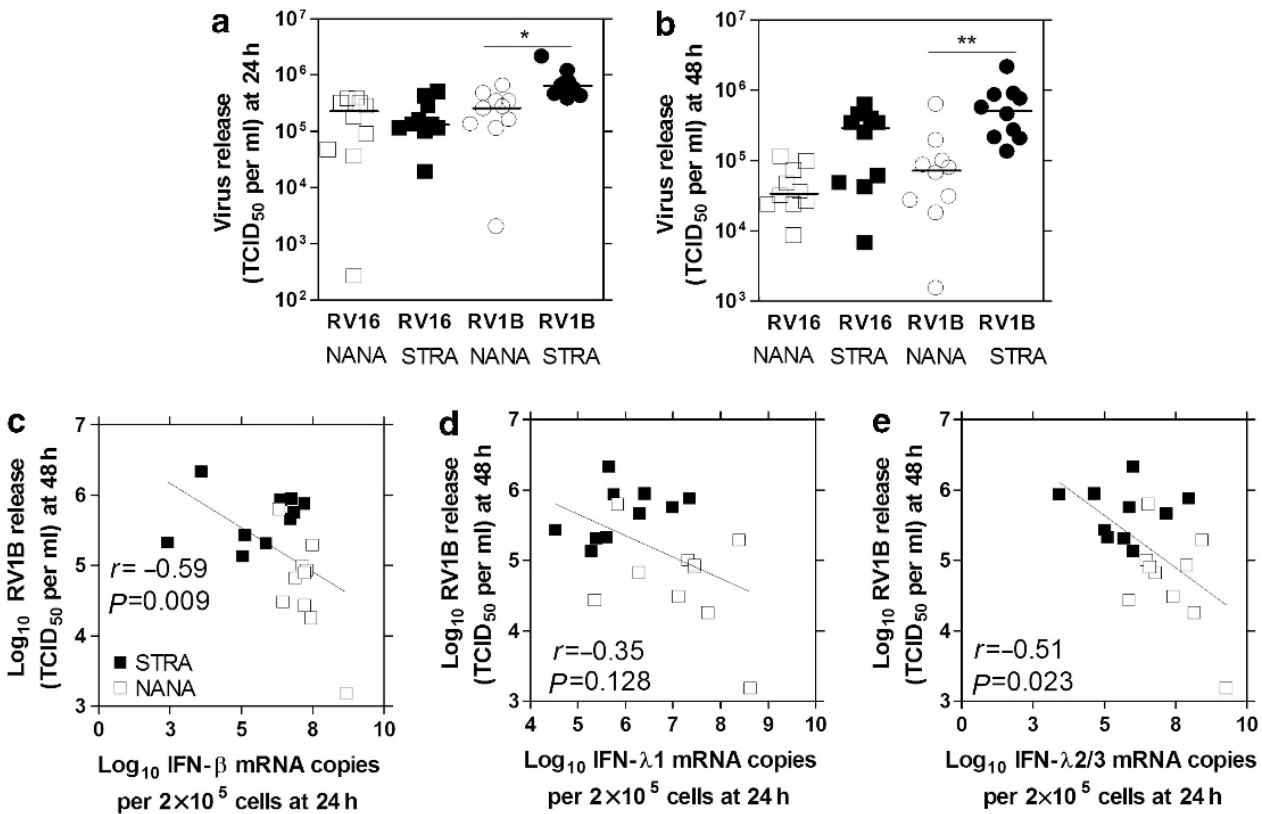

Figure 4 Bronchial epithelial cells (BECs) from severe therapy resistant asthma (STRA) have increased RV load, which negatively correlated with interferon (IFN) induction. Non-atopic non-asthmatic (NANA) $(n=10)$ and STRA $(n=10)$ BEC cultures were infected with RV16, RV1B, or medium and RV load (TCID 50 per $\mathrm{ml}$ ) measured at (a) 24 or (b) $48 \mathrm{~h}$ post-infection. RV1B load at $48 \mathrm{~h}$ was then correlated with RV1B induced (c) IFN- $\beta$, (d) IFN- $\lambda 1$, and (e) IFN- $\lambda 2 / 3$ mRNA at $24 \mathrm{~h}$. Horizontal line indicates the median ${ }^{*} P<0.05,{ }^{\star \star} P<0.01$ vs. medium or as indicated.

and $P<0.01$, respectively), but not TLR3 (Figure 3c). In STRA samples, RIG-I, MDA5 and TLR3 was not significantly induced by either virus. STRA BECs had significantly lower RIG-I mRNA in RV16 (9.6-fold difference vs. control, $P<0.01)$ and RV1B (9.2-fold difference, $P<0.05)$ infected cultures, significantly lower MDA5 also for RV16 (7.3-fold difference, $\quad P<0.05)$ and RV1B $\quad$ 5.8-fold difference, $P<0.05)$ infected cultures, and significantly lower TLR3 mRNA in RV16 (11-fold difference, $P<0.001$ ) and RV1B (16.6-fold difference, $P<0.01)$ infected cultures, and also lower TLR3 levels in cells treated with medium only (3.4-fold difference, $P<0.01$ ) (Figures 3a-c).

\section{STRA BECs exhibited increased RV load that negatively correlated with IFN- $\beta$ and IFN- $\lambda$ expression}

We next examined RV release, hypothesizing that differences in IFN expression between NANA and STRA should lead to differences in RV release with STRA likely having higher RV release due to impaired IFN induction. Changes in RV load would also be consistent with the idea that IFN protein was being produced by the epithelial cells, and induce IFNmediated signaling and thus ISGs that affect virus growth. We found that abundance of RV1B but not RV16 was significantly higher at $24 \mathrm{~h}$ post-infection in STRA compared with NANA $(P<0.05)$, with the median value for STRA for RV16 being $<1$-fold lower than NANA, but 2.5-fold higher for RV1B (Figure 4a). At 48 h, RV1B STRA virus load was significantly higher still, when compared with NANA (7-fold higher median, $P<0.01$ ) while RV16 virus release showed a trend for higher levels in STRA (8.7-fold higher median) but was not statistically significant (Figure $\mathbf{4 b}$ ). Correlations showed that
RV1B induced IFN- $\beta$ and IFN- $\lambda 2 / 3$ mRNA levels at $24 \mathrm{~h}$ negatively correlated with RV1B loads at $48 \mathrm{~h}$ (Figure $4 \mathrm{c}$ and e) and there was a trend for a negative correlation for RV1B induced IFN- $\lambda 1$ mRNA levels (Figure 4d). We did not observe any significant correlations with any IFN mRNA at $24 \mathrm{~h}$ and RV16 load at $48 \mathrm{~h}$ (data not shown).

\section{STRA BECs have reduced IFN- $\lambda$ protein at $24 \mathrm{~h}$ compared} with NANA

RV16 and RV1B induced IFN- $\beta$, IFN- $\lambda$ and CXCL8 protein in culture supernatants at $24 \mathrm{~h}$ was also measured. Expression of IFN- $\beta$ was barely detectable at $24 \mathrm{~h}$, not significantly induced by either RV compared with medium at $24 \mathrm{~h}$, but levels were significantly greater in NANA than in STRA for RV16 $(P<0.05)$ but not for RV1B (Figure 5a). IFN- $\lambda$ levels were more robustly induced by RV16 and RV1B, but not significantly different from medium. IFN- $\lambda$ protein was significantly lower for STRA compared with NANA for both RV16 $(P<0.01)$ and RV1B $(P<0.05) \quad$ (Figure 5b). CXCL8 showed trends for induction by RV16 and RV1B in NANA that was not significantly different from medium treated cells. RV1B, but not RV16 induced CXCL8 was significantly induced compared with medium for STRA BECs $(P<0.01)$. There was no significant difference between STRA and NANA; however, STRA showed trends for lower levels of CXCL8 release (Figure 5c). We could not detect CXCL5 in amounts greater than the sensitivity of the ELISA (data not shown).

STRA BECs have deficient IFN mRNA abundance upon stimulation with polylC at $8 \mathrm{~h}$

We next assessed the expression of IFN $-\beta$, IFN- $\lambda 1$, IFN- $\lambda 2 / 3$ mRNA following stimulation with the TLR3 ligand, polyIC at 

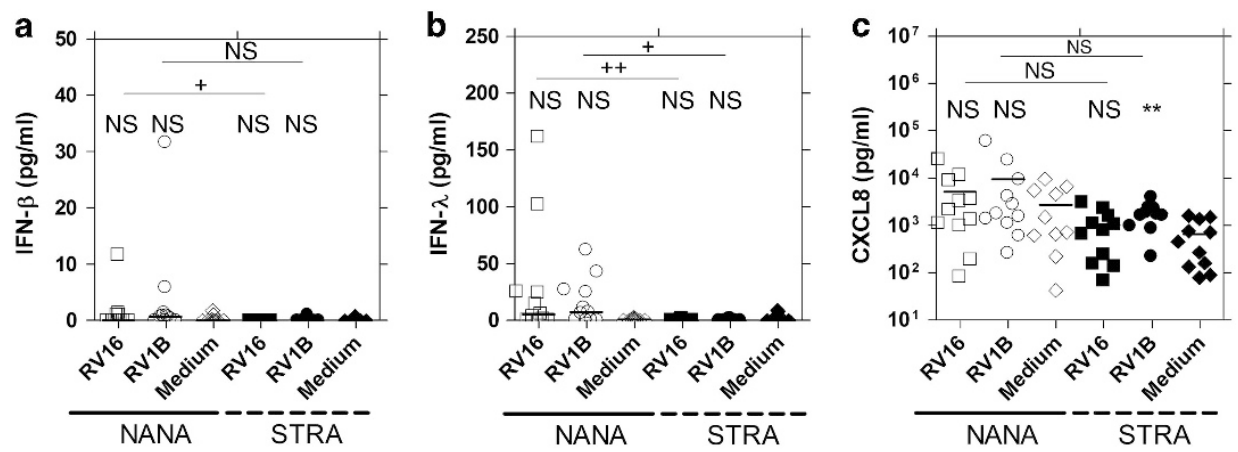

Figure 5 Bronchial epithelial cells (BECs) from severe therapy resistant asthma (STRA) have impaired RV16 and RV1B induced interferon (IFN)- $\beta$, and IFN- $\lambda$ protein in supernatants at $24 \mathrm{~h}$ compared with non-atopic non-asthmatic (NANA). NANA $(n=11)$ and STRA ( $n=11)$ BEC cultures were infected with RV16, RV1B or medium and (a) IFN- $\beta$, (b) IFN- $\lambda 1$, and pro-inflammatory cytokine (c) CXCL8 protein release in supernatants measured at $24 \mathrm{~h}$ post-infection. Horizontal line indicates the median ${ }^{* \star} P<0.01$ vs. medium, ${ }^{+} P<0.05,{ }^{++} P<0.01$, as indicated, NS $=$ not significant.
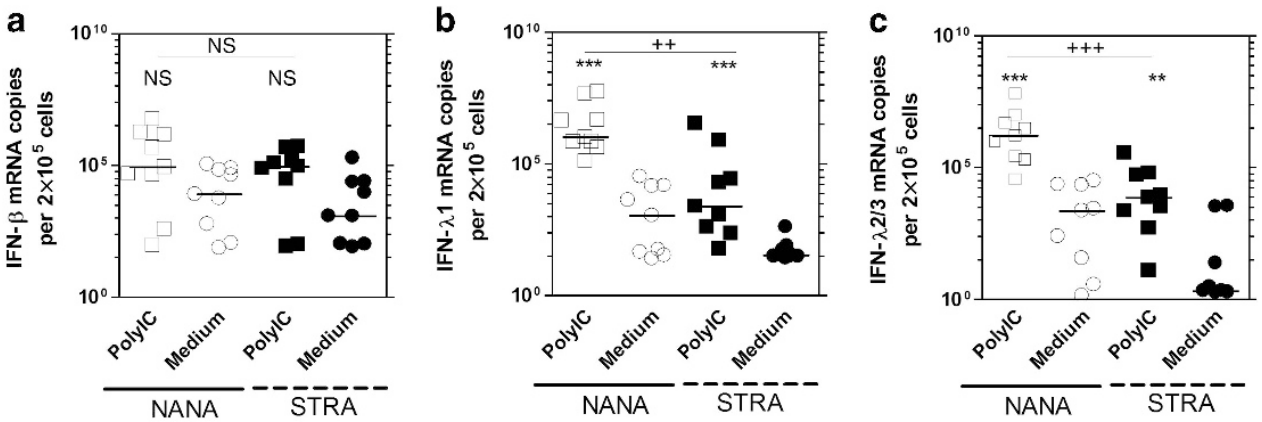

Figure 6 Bronchial epithelial cells (BECs) from severe therapy resistant asthma (STRA) have impaired polylC induced interferon (IFN)- $\lambda 1$ and IFN- $\lambda 2 / 3$ mRNA compared with non-atopic non-asthmatic (NANA) at 8 h. NANA $(n=9)$ and STRA $(n=9)$ BEC cultures were treated with $1 \mu \mathrm{g} / \mathrm{ml}$ polylC or medium and (a) IFN- $\beta$, (b) IFN- $\lambda 1$, (c) IFN- $\lambda 2 / 3$ mRNA measured at $8 \mathrm{~h}$ post-infection. Horizontal line indicates the median. ${ }^{* \star} P<0.01$, ${ }^{* \star \star} P<0.001$ vs. medium, ${ }^{+}+P<0.01$, as indicated, NS $=$ not significant.

$8 \mathrm{~h}$, as previously reports of asthmatic BECs have observed deficient IFN responses using polyIC. ${ }^{12,14}$ Previously, studies in adults showed that the peak IFN mRNA expression with polyIC is at $8 \mathrm{~h}$, so differences at $8 \mathrm{~h}$ between NANA and STRA were examined. At $8 \mathrm{~h}$ post-infection, we did not observe significant induction of IFN- $\beta$ in NANA or STRA, by polyIC, and no significant difference in IFN- $\beta$ mRNA abundance between the two groups (fold difference in median of $<1$ ) (Figure 6a). For IFN- $\lambda 1$, we observed a significant induction by polyIC for NANA $(P<0.001)$ and STRA $(P<0.001)$, and STRA had significantly less IFN- $\lambda 1 \mathrm{mRNA}$ abundance when compared with NANA (fold difference in median of $433, P<0.01$ ) (Figure 6b). For IFN- $\lambda 2 / 3$, we observed a significant induction by polyIC in NANA $(P<0.001)$ and STRA $(P<0.01)$, with STRA having significantly less IFN $-\lambda 2 / 3$ mRNA than NANA (fold difference in median of 218, $P<0.001$ ) (Figure 6c). We also measured the induction of two non-IFN genes, CXCL8 and CXCL5, and compared expression levels in NANA and STRA. We observed that CXCL8 and CXCL5 mRNA abundance was not significantly induced by polyIC vs. medium treated controls at $8 \mathrm{~h}$ and no differences were observed between NANA and STRA (data not shown).
STRA BECs exhibited impaired TLR3 mRNA levels in unstimulated cells and unimpaired polyIC induced RIG-I and MDA5 mRNA abundance at $8 \mathrm{~h}$

We also wanted to assess if polyIC failed to induce the pattern recognition receptors in STRA BECs. Previously, we established that both RIG-I and MDA5 are polyIC inducible in BECs. ${ }^{18} \mathrm{At}$ $8 \mathrm{~h}$ post-treatment, we found that polyIC significantly induced RIG-I mRNA in NANA $(P<0.01)$ and STRA BECs $(P<0.05)$ (Figure 7a), and also induced MDA5 in NANA $(P<0.05)$ but not STRA BECs (Figure 7b). We also observed that polyIC significantly induced TLR3 mRNA in NANA $(P<0.05)$ but not STRA BECs (Figure 7c). When comparing mRNA induction of the receptors between NANA and STRA BECs, we observed no significant difference for RIG-I, or MDA5 but found that medium treated and polyIC induced TLR3 mRNA was lower in STRA BECs $(P<0.05,3.6$-fold difference; and 4.7-fold difference $P<0.01$, respectively) (Figure 7c).

\section{STRA BECs exhibit reduced polyIC induced IFN- $\lambda$ and} CXCL8 protein release compared with NANA

PolyIC induced IFN- $\beta$, IFN- $\lambda$, and CXCL8 protein in culture supernatants was also measured at 8 and $24 \mathrm{~h}$. At $8 \mathrm{~h}$, no 

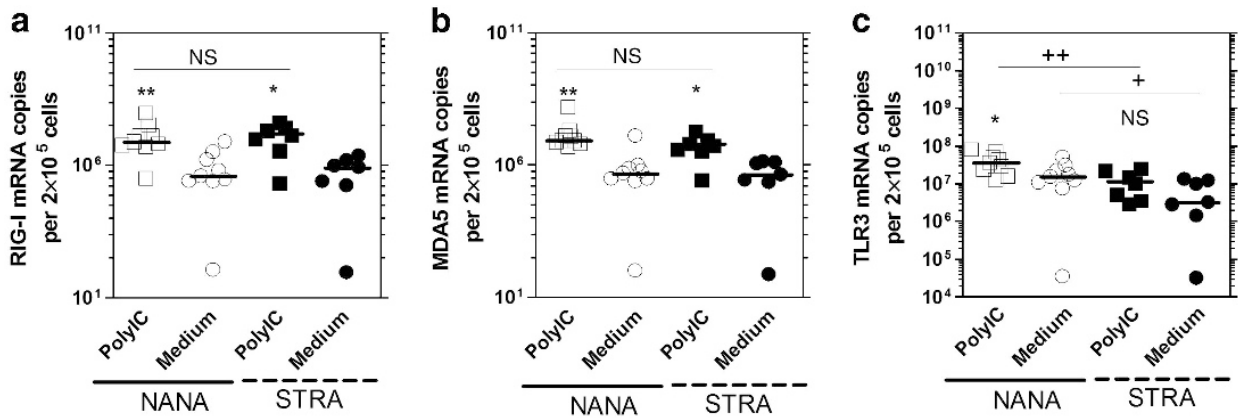

Figure 7 Unstimulated bronchial epithelial cells (BECs) from severe therapy resistant asthma (STRA) have impaired Toll-like receptor-3 (TLR3) mRNA levels but not impaired polyIC induced RIG-I and MDA5 mRNA compared with non-atopic non-asthmatic (NANA) at $8 \mathrm{~h}$. NANA $(n=9)$ and STRA $(n=7)$ BEC cultures were treated with $1 \mu \mathrm{g} / \mathrm{ml}$ polyIC or medium and (a) RIG-I (b) MDA5 (c) TLR3 mRNA measured at $8 \mathrm{~h}$ post-infection. Horizontal line indicates the median. ${ }^{*} P<0.05,{ }^{* *} P<0.01$ vs. medium, ${ }^{+} P<0.05,{ }^{+}+P<0.01$, as indicated, $N S=$ not significant.
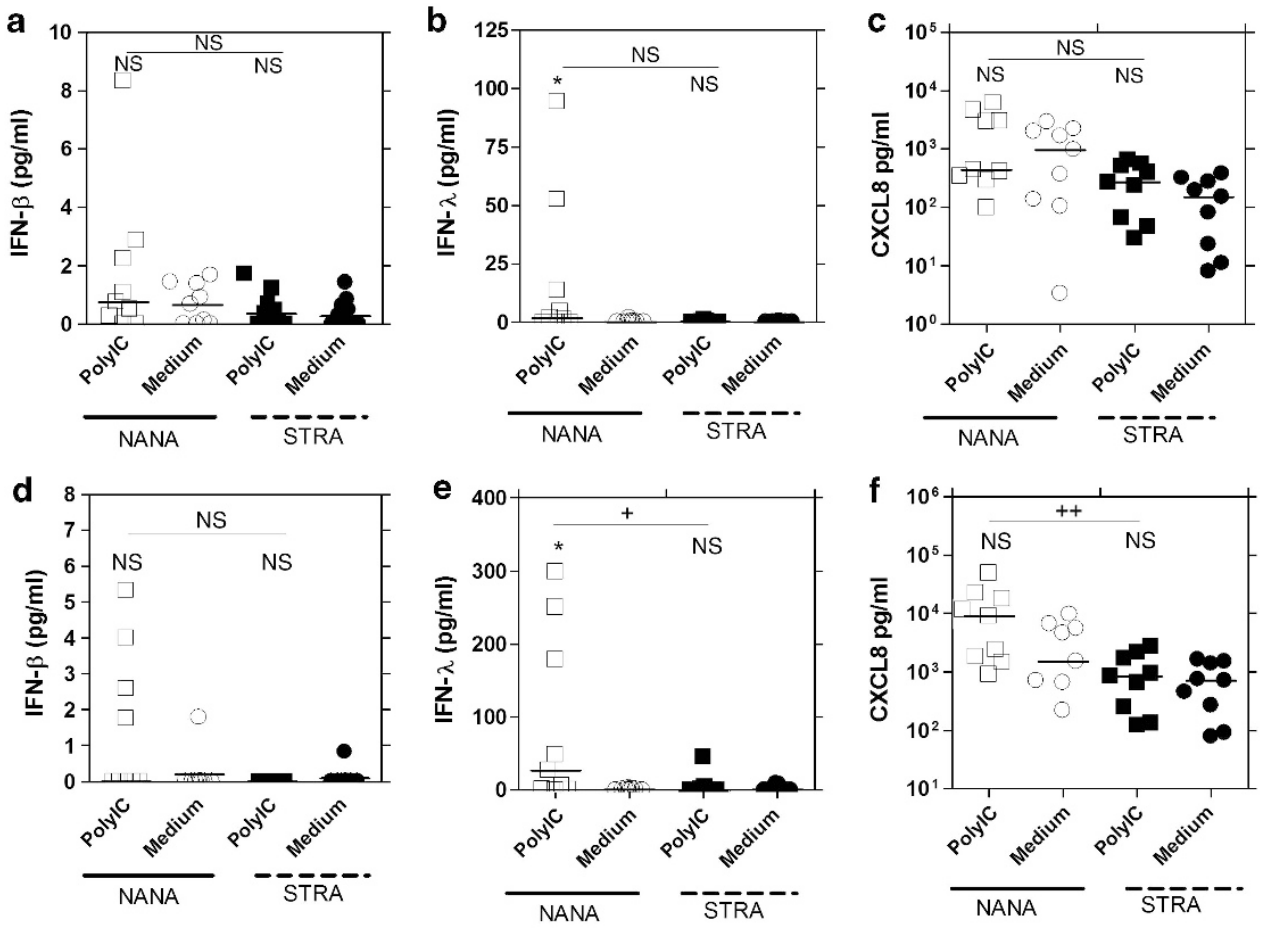

Figure 8 Bronchial epithelial cells (BECs) from severe therapy resistant asthma (STRA) have impaired polyIC induced interferon (IFN)- $\beta$, IFN- $\lambda$ and CXCL8 protein in supernatants compared with non-atopic non-asthmatic (NANA) at $24 \mathrm{~h}$. NANA $(n=9)$ and STRA $(n=9)$ BEC cultures were treated with $1 \mu \mathrm{g} / \mathrm{ml}$ polylC or medium and (a) IFN- $\beta$, (b) IFN- $\lambda 1$, (c) CXCL8 protein release in supernatants measured at $8 \mathrm{~h}(\mathbf{a}-\mathbf{c})$ and $24 \mathrm{~h}$ (d-f) post-infection. Horizontal line indicates the median ${ }^{*} P<0.05$ vs. medium ${ }^{+} P<0.05,{ }^{+}+P<0.01$, as indicated, NS $=$ not significant.

significant difference in IFN- $\beta$ (Figure 8a) or IFN- $\lambda$ (Figure 8b) was observed between NANA and STRA, although NANA showed trends for a greater IFN- $\lambda$ response. CXCL8 protein was not significantly induced in NANA and STRA but showed trends for greater CXCL8 levels upon polyIC treatment. There was no significant difference between NANA and STRA, although STRA showed trends for lower levels of CXCL8 (Figure 8c). At $24 \mathrm{~h}$ post-treatment, IFN- $\beta$ was again not significantly induced in NANA or STRA BECs and not significantly different between the two groups but more IFN- $\beta$ was seen in NANA (Figure 8d). For IFN- $\lambda$, NANA showed significant induction by polyIC $(P<0.05)$; however, STRA did not, and NANA IFN- $\lambda$ levels were significantly higher than for STRA $(P<0.05)$ (Figure 8e). For CXCL8, NANA and STRA showed no significant induction by polyIC, exhibited trends for increased CXCL8 release upon polyIC treatment for both groups and CXCL8 levels were significantly lower in STRA stimulated BECs compared with NANA BECs $(P<0.01)$ (Figure 8f). CXCL5 was not detectable in culture supernatants following polyIC treatment (data not shown).

IFN mRNA levels from STRA BECs were not associated with IgE, markers of atopy ICS, number of exacerbations and lung function

We also attempted to correlate IFN mRNA levels at $24 \mathrm{~h}$ and virus load data at $48 \mathrm{~h}$ with total IgE, numbers of positive RAST 
tests, cumulative RAST tests, BAL and sputum neutophil and eosinophil numbers inhaled corticosteroids (ICS), numbers of exacerbations and lung function. We found no correlation with any IFN mRNA or virus load with any of these clinical end points (data not shown).

\section{DISCUSSION}

In this study we have identified that BECs from STRA children have impaired innate IFN responses to RV infection and polyIC stimulation. Impaired IFN expression was related to increased virus load, yet unrelated to age, IgE, or lung function. This study has confirmed impaired innate responses to RV in asthmatic BECs, ${ }^{12-14}$ and are important for a further two reasons; this is the first observation in severe asthma and confirms a previous report $^{11}$ investigating impaired IFN responses in BECs from asthmatic children, including children as young as 9 years of age.

The strong associations between virus infections in asthma onset early in life and as precipitants of asthma exacerbations later in life has led to the hypothesis that asthmatics may have impaired protective immune responses to viruses and that increased virus replication may lead to greater duration and severity of asthma symptoms. ${ }^{3}$ Evidence for this idea came with later studies of innate IFN production in mild-moderate asthmatic BECs cultured ex vivo ${ }^{12,13}$ which showed that asthmatic cells have a marked deficiency in IFN- $\beta$ and IFN- $\lambda$ production. Since, there has been one study in mixed moderate and severe asthmatic adult donors treated with polyIC ${ }^{14}$ but no studies have yet investigated severe asthma exclusively. Two recent studies did not observe impaired IFN expression in studies of mild-moderate asthmatics, ${ }^{22,26}$ suggesting that these observations may be restricted to a poorly defined subgroup. We therefore set out to examine if STRA children would also exhibit impaired IFN production. The causes of STRA are incompletely understood, and severe asthmatics present an important subgroup of asthma in need of new therapies. If these patients too showed evidence of defective anti-viral immunity, this could lead to new therapeutic options for severe asthma such as IFN- $\beta$ treatment. ${ }^{31}$ The identification of impaired IFN responses in children, including very young children could provide important insights into the mechanism(s) responsible for this defect. We have thus extended the original studies and report that BECs derived from STRA children have profound defects in IFN- $\beta$ and IFN- $\lambda$ production, and therefore show elevated RV replication.

Our STRA children all experienced persistent symptoms despite maximal therapy. We therefore, cannot rule out that some of our data may be influenced by therapy. It is important to point out, however, that BEC cultures are often seeded by a few initial cells obtained in the brushings, which take $\sim 2-3$ weeks to grow before being used for experiments. Therefore, it is difficult to argue that the experimental BECs themselves are influenced by therapy, as they have not been exposed to it. In support we saw no association between ICS usage and IFN abundance or RV replication. We cannot also distinguish between the effects of atopy vs. asthma, as all our children were atopic asthmatics, our controls are, however, healthy NANAs. Further studies are required to make this important distinction. One strength of our study is that all STRA children are truly therapy resistant, as non-adherent children are careful filtered out of the study group during this protocol. We experienced great difficulty in recruiting appropriate STRA children and controls, and spent over 3 years recruiting 11 STRA children and NANA controls. Performing bronchoscopes and obtaining brushings in children is technically difficult, and is not commonly performed, as supported by the few published studies available in the literature. ${ }^{32-34}$ Controls can be especially difficult to recruit, thus limiting overall numbers. This study represents the first investigation of impaired innate responses to viruses in STRA.

We have shown that IFN- $\beta$, IFN- $\lambda 1$, and IFN- $\lambda 2 / 3$ mRNA are impaired in asthmatic BECs, and this is specific for IFNs, as the NF- $\kappa B$ regulated pro-inflammatory cytokines CXCL8 and CXCL5 mRNA were very similar between NANA and STRA, although polyIC induced CXCL8 protein release was significantly higher in NANA. The decreased CXCL8 protein, a pro-inflammatory cytokine, observed in STRA does not fit with the mRNA data or what has been previously seen in asthmatic BEC studies ${ }^{12}$ or BAL macrophages that exhibited deficient RV induced IFN. Also, in a recent study in cystic fibrosis (CF), BECs from CF children exhibited impaired IFN expression after infection with RV but again had comparable CXCL8 levels to controls. ${ }^{35}$ The differences in CXCL8 protein levels between NANA and STRA in the present study may have been influenced by a few NANA individuals who also exhibited unusually increased spontaneous CXCL8 release in uninfected, medium treated cells. Larger numbers are required to identify if reduced CXCL8 in STRA is reproducible or is an anomaly of our study.

We found it difficult to measure released IFN protein, especially IFN- $\beta$ in our culture supernatants. IFN- $\beta$ protein is notoriously difficult to measure and may be unstable. Hence, our results for IFN- $\beta$ protein measurements may not truly reflect how deficient this protein is in STRA. IFN- $\lambda$ was produced at higher levels and was easier to measure, and in general results showed that IFN- $\lambda$ protein was impaired in STRA. As surrogate markers of IFN protein production, we measured RV replication and RLH mRNA expression. Again there were distinct differences between RLH mRNA induction and RV replication in STRA vs. controls, indirectly suggesting that IFN protein levels are different in these two populations. We found that RV1B but not RV16 negatively correlated with IFN mRNA levels, and why RV1B but not RV16 gave this association is difficult to explain. RV1B replicated at higher levels and produced more IFN, it is possible that this made associations to be more readily observed. It is also possible that each virus responds subtly differently to IFN, affecting the chances of observing meaningful associations between the two.

There are several possible ways in which defective IFN- $\beta$ and/or IFN- $\lambda$ could impact on asthma exacerbation pathogenesis. Herein, we observed increased RV release in STRA BECs that was negatively related to IFN levels produced in the same 
cells, suggesting that an inability to control early virus replication and dissemination in the lower airway by having impaired IFN (but not impaired virus induced inflammation) likely leads to later epithelial destruction, enhanced virus induced inflammation, ${ }^{2,36}$ and mucus hyper secretion, ${ }^{37}$ and therefore has enhanced negative effects on lung function. In support, the previous study by Contoli showed that defective IFN- $\lambda$ and IFN- $\lambda 2 / 3$ was related to enhanced cold symptoms, airway inflammation, and decreases in lung function. ${ }^{13}$ The IFN-inducible cytokine IL-15 has also been inversely related to increased cold score and airway hyper responsiveness in asthmatics. ${ }^{38}$ A recent study has highlighted the anti-Th2 nature of IFN- $\lambda$ in a mouse model, suggesting that IFNs may be antagonists of Th2 immunity. ${ }^{39}$ Furthermore, asthmatic pDCs have impaired IFN- $\alpha$ responses following influenza challenge, which is negatively related to serum IgE; and cross-linking Fc\&R1-bound IgE reduced IFN- $\alpha$ induction in response to influenza ${ }^{30}$ suggesting again that Th2 pathways and innate IFN responses reciprocally regulate each other. Thus, there are many mechanisms through which a defective IFN- $\beta$ or IFN- $\lambda$ response may promote virus induced asthma exacerbations.

In our study of STRA children, we could not find any relationship between atopy and allergy (RAST, IgE levels) or Th2-mediated inflammation (eosinophils) with impaired IFN. We also investigated associations between ICS usage and numbers of exacerbations, and found no associations. This could be due to the effects of therapy skewing these clinical markers of disease such that the true levels are diluted, or possibly due to the small sample size employed. Alternatively, other data show that STRA is a disease not strongly associated with Th2 cytokines such as IL-4 and IL- $13^{10}$ such that important clinical associations with impaired IFN in STRA may not be associated with atopy, allergy, or Th2 immunity. Further larger studies are required to identify clinical outcomes that may be associated with impaired IFN in STRA.

Recently, the signaling pathway(s) used by RV to induce IFN- $\beta$ and IFN- $\lambda$ have been studied. ${ }^{17,18,40} \mathrm{RV}$ dsRNA is recognized by TLR 3 and the RLHs RIG-I and MDA5. The bulk of the evidence thus far does not support that a direct germline defect in these pathway(s) may explain impaired IFN in asthma. Array studies ${ }^{22}$ have not found that these pattern recognition receptors are poorly expressed in asthma, and large genotyping studies have not highlighted any single-nucleotide polymorphisms associated with asthma for these genes. ${ }^{41,42}$ These receptors also activate signaling pathways that are also common for pro-inflammatory cytokines, ${ }^{18}$ which are not consistently reduced in studies where impaired IFN are observed. ${ }^{12,14}$ Of interest we found less TLR3 mRNA in untreated STRA cells vs. controls. These data suggest that TLR3 expression may be impaired in asthmatic cells, but this observation was not seen in a recent study of asthmatic BAL macrophages. ${ }^{27}$ The decreased expression of TLR3 in STRA cells may also explain trends for lower CXCL8 protein release, as RV induction of CXCL8 is TLR3 dependent. ${ }^{18}$ We also saw decreased induction of RIG-I and MDA 5 by viruses at $24 \mathrm{~h}$, which we believe is due to a lack of IFN acting as an additional inducer of RIG-I and MDA5. ${ }^{18}$
Curiously, differences between STRA and NANA were not seen for polyIC after $8 \mathrm{~h}$, presumably because IFN had not had time to signal back and contribute to further RLH induction at this early time point. Whether or not defective IFN in severe asthma is due to germline defects in a pattern recognition receptor or signaling molecule in TLR or RLH pathway requires further exploration.

In summary, we have shown for the first time that impaired IFN- $\beta$ and IFN- $\lambda$ induction is a feature of BECs cultured from STRA children. There is also a functional readout; STRA BECs also have higher RV loads that are consistent with impaired IFN expression. We therefore conclude that impaired innate IFN expression is a feature of STRA and can be observed in young children. This study has therefore extended existing knowledge concerning impaired innate immunity in asthma and could potentially impact on how STRA and virus induced exacerbations of STRA are managed in the future.

\section{METHODS}

Severe therapy resistant asthmatic (STRA) and NANA children. BECs were obtained from 11 pediatric patients with STRA undergoing clinically indicated bronchoscopies. STRA was defined as persistent (most days, for at least 3 months) chronic symptoms of airway obstruction (requiring a rescue bronchodilator on at least 3 days per week), despite treatment with high dose ICSs (at least $800 \mu \mathrm{g} /$ day of beclomethasone equivalent) and trials of add on drugs (long acting $\beta_{2}$ agonists, leukotriene receptor antagonists, and oral theophylline in a low, anti-inflammatory dose) and/or recurrent severe asthma exacerbations. ${ }^{43}$ None of the subjects was taking antibiotics at the time of the study and all were considered to be free of clinical infections. All had been evaluated in detail to exclude reversible factors such as poor adherence as a cause of their severe asthma. ${ }^{44,45}$ Eleven children (NANA) with no history of asthma, no record of food allergy, rhinitis or eczema, and no family history of asthma were recruited. After written consent, these children were recruited into the study while being intubated for other clinical procedures (including haemoptysis, laryngomalacia, and congenital cystic adenomatoid malformation). Approval for the study of pediatric patients was given by Royal Brompton Hospital Ethics Committee, and the Ethics Committee of the Canton of Bern, Switzerland. Written informed consent from parents and age-appropriate assent form children was obtained in each case. Table 1 provides the details of the participants in the study.

BEC culture. BECs were grown from brushings (up to 6 brushes per patient) of the bronchial tree during bronchoscopy or through the endotracheal tube in subjects undergoing elective surgery under general anesthesia. BECs were cultured according to previously published protocols in BEGM medium (Clonetics, Basal Switzerland). ${ }^{12,32}$ BECs were split when confluent, and seeded for experimentation at passage 2 . Analysis by light microscopy showed that cultures had the typical cobblestone appearance of epithelial cells and staining for cytokeratin-19 confirmed the epithelial nature of all cultures (Supplementary Figure S1 online).

Virus culture, reagents, and treatment of BECs. RV serotypes 16 (major group) and 1B (minor group) were grown in HeLa cells as previously described. ${ }^{18}$ PolyIC was purchased from Sigma-Aldrich (Dorset, UK), made up at $1 \mathrm{mg} / \mathrm{ml}$ in water and stored at $-80^{\circ} \mathrm{C}$. Primary BECs obtained from STRA and NANA donors were infected with RV1B or RV16 for $1 \mathrm{~h}$ as previously described. ${ }^{12}$ PolyIC was diluted in BEBM medium at $1 \mu \mathrm{g} / \mathrm{ml}$ and incubated with shaking for $1 \mathrm{~h}$ at room temperature, medium was then replaced and cells harvested at various time points. 
Table 1 Characteristics of NANA and STRA volunteers

\begin{tabular}{lccc}
\hline Parameter & NANA & STRA & $P$-value \\
\hline$n$ & 11 & 11 & \\
Age & $7(2-$ & $11(9-15)$ & $0.11^{\mathrm{a}}$ \\
& $15)$ & & \\
\% Male sex & $64 \%$ & $55 \%$ & $1.0^{\mathrm{b}}$ \\
Inhaled corticosteroids ( $\mu \mathrm{g} /$ day) & ND & $1,600(800-4,000)$ & \\
No. of exacerbations in 6 & ND & $3(0-6)$ & \\
months & & & \\
Family history of atopy & No & Yes \\
lgE (IU/ml) & ND & $374(121-4,610)$ & \\
No. + ve RAST tests & ND & $3(0-6)$ & \\
Cumulative RAST score & ND & $60.37(0.025-195)$ & \\
Family history of asthma & No & Yes \\
Physician diagnosis of asthma & No & Yes \\
FEV1 (\% predicted) & ND & $79(36-108)$ \\
FVC (\% predicted) & ND & $80.6(19-111)$ & \\
\hline
\end{tabular}

FVC, forced vital capacity; NANA, non-atopic non-asthmatic; STRA, severe therapy resistant asthma.

Data are given as median (range) unless otherwise stated. ND = no data.

aDetermined by Mann-Whitney $U$ test.

DDetermined by Fisher's exact test. "RAST tests include house dust mite, mixed grasses, dog, cat, egg white, peanut, and mixed tree pollen, Aspergillus fumigatus, Alternaria sp, Cladosporium herbarum, mouse and cockroach allergens. Cumulative RAST tests are the sum of all RAST scores. FEV1 forced expiratory volume in one second.

RNA extraction and quantitative RT-PCR. Total RNA was extracted using commercially available reagents (Qiagen, Crawley, UK) according to the manufacturer's recommended protocol. cDNA was synthesized (Qiagen) and $1 \mu \mathrm{l}$ used as template in quantitative PCR (ABI Biosystems, Paisley, UK). NANA and STRA samples were run together on each plate, to minimize the effects of inter-assay variation on differences between the two groups. The following genes were quantified by comparison to standard curves using plasmid DNA, IFN- $\beta$, IFN- $\lambda 1$, IFN- $\lambda 2 / 3$, TLR3, RIG-I, MDA5, IL-8 (CXCL8), and ENA-78 (CXCL5) using 18S rRNA as a template control. All primers and probe sequences are previously reported and were used in ratios as previously described. ${ }^{18,27}$

Assessment of RV load by titration on HeLa cells. Supernatants were thawed and used to infect HeLa cells as previously described. ${ }^{46}$ The amount of RV16 or RV1B was assessed as a TCID 50 value using the Karber formula. ${ }^{47}$

Analysis of IFNs and cytokines by ELISA. IFN and cytokine protein was assessed in sample supernatants using ELISA using reagents from R\&D Systems (Abingdon, UK) according to the manufacturer's recommended protocol. Sensitivities were IFN- $\beta ; 1 \mathrm{pg} / \mathrm{ml}$, IFN- $\lambda$; $15 \mathrm{pg} / \mathrm{ml}$, and IL-8; $15 \mathrm{pg} / \mathrm{ml}$.

Statistics. Power calculations performed in our group show that $n=11$ adult subjects per group is large enough to detect up to threefold differences in $95 \%$ of mRNAs detected by qPCR. There are no available data on children to perform power calculations. As data were not normally distributed, non-parametric statistics were employed. The Kruskal-Wallis test was first employed in each data set; if significant differences were identified between two groups by pin pointing using the Mann-Whitney $U$ test, with $P<0.05$ taken as significant.

SUPPLEMENTARY MATERIAL is linked to the online version of the paper at http://www.nature.com/mi

\section{ACKNOWLEDGEMENTS}

We thank Angela Fonceca and Dr Paul McNamara for advice and guidance on growing BECs. MRE was supported by a Fellowship and SLJ by a Chair from Asthma UK (RF07_04, CH11SJ, respectively). This work was supported in part by grants from the British Lung Foundation (P06/13), The Ulrich Müller-Gierok Foundation (to NR), MRC project Grant G0601236, MRC Center Grant G1000758, ERC FP7 Advanced Grant 233015 (to SLJ), Predicta FP7 Collaborative Project Grant 260895 and the Wellcome Trust sponsored Centre for Respiratory Infection (CRI) (Wellcome Trust Strategic Award 083567/Z/07/Z) the NIHR Respiratory Disease Biomedical Research Unit at the Royal Brompton and Harefield NHS Foundation Trust the NIHR Biomedical Research Centre at Imperial College London and the NIHR Clinical Lecturers funding scheme. SLJ is an author on patents regarding the use of interferon therapies for treatment of exacerbations of airway disease and holds share options in Synairgen who are a company developing interferon therapies for treatment of exacerbations of airway disease.

\section{DISCLOSURE}

The authors declared no conflict of interest.

c) 2013 Society for Mucosal Immunology

\section{REFERENCES}

1. Johnston, S.L. et al. Community study of role of viral infections in exacerbations of asthma in 9-11 year old children. BMJ 310, 1225-1229 (1995).

2. Message, S.D. et al. Rhinovirus-induced lower respiratory illness is increased in asthma and related to virus load and Th1/2 cytokine and IL-10 production. Proc. Natl. Acad. Sci. USA 105, 13562-13567 (2008).

3. Corne, J.M. et al. Frequency, severity, and duration of rhinovirus infections in asthmatic and non-asthmatic individuals: a longitudinal cohort study. Lancet 359, 831-834 (2002).

4. Johnston, N.W. et al. The September epidemic of asthma exacerbations in children: a search for etiology. J. Allergy Clin. Immunol. 115, 132-138 (2005).

5. Grunberg, K. et al. Rhinovirus-induced airway inflammation in asthma: effect of treatment with inhaled corticosteroids before and during experimental infection. Am. J. Respir. Crit. Care Med. 164, 1816-1822 (2001).

6. Pauwels, R.A. et al. Effect of inhaled formoterol and budesonide on exacerbations of asthma. Formoterol and Corticosteroids Establishing Therapy (FACET) International Study Group. N. Engl. J. Med. 337, 14051411 (1997).

7. Pauwels, R.A. et al. Early intervention with budesonide in mild persistent asthma: a randomised, double-blind trial. Lancet 361, 1071-1076 (2003).

8. FitzGerald, J.M. et al. Doubling the dose of budesonide versus maintenance treatment in asthma exacerbations. Thorax 59, 550-556 (2004).

9. Bel, E.H. et al. Diagnosis and definition of severe refractory asthma: an international consensus statement from the Innovative Medicine Initiative (IMI). Thorax 66, 910-917 (2011).

10. Bossley, C.J. etal. Pediatric severe asthma is characterized by eosinophilia and remodeling without $\mathrm{T}(\mathrm{H}) 2$ cytokines. J. Allergy Clin. Immunol. 129, 974-82.e13 (2012).

11. Baraldo, S. et al. Deficient antiviral immune responses in childhood: distinct roles of atopy and asthma. J. Allergy Clin. Immunol. (2012) (in press).

12. Wark, P.A. et al. Asthmatic bronchial epithelial cells have a deficient innate immune response to infection with rhinovirus. J. Exp. Med. 201, 937-947 (2005).

13. Contoli, M. et al. Role of deficient type III interferon-lambda production in asthma exacerbations. Nat. Med. 12, 1023-1026 (2006).

14. Uller, L. et al. Double-stranded RNA induces disproportionate expression of thymic stromal lymphopoietin versus interferon-beta in bronchial epithelial cells from donors with asthma. Thorax 65, 626-632 (2010).

15. likura, K. et al. Peripheral blood mononuclear cells from patients with bronchial asthma show impaired innate immune responses to rhinovirus in vitro. Int Arch. Allergy Immunol. 155 (Suppl 1), 27-33 (2011). 
16. Khaitov, M.R. [Acute respiratory viral infections and bronchial asthma. Cellular and molecular aspects of the problem]. Zh Mikrobiol. Epidemiol. Immunobiol., 84-93 (2002).

17. Wang, Q. et al. Role of double-stranded RNA pattern recognition receptors in rhinovirus-induced airway epithelial cell responses. J. Immunol. 183, 6989-6997 (2009).

18. Slater, L. et al. Co-ordinated role of TLR3, RIG-I and MDA5 in the innate response to rhinovirus in bronchial epithelium. PLoS Pathog. 6, e1001178 (2010).

19. Kotenko, S.V. et al. IFN-lambdas mediate antiviral protection through a distinct class II cytokine receptor complex. Nat Immunol 4, 69-77 (2003).

20. Meager, A., Visvalingam, K., Dilger, P., Bryan, D. \& Wadhwa, M. Biological activity of interleukins-28 and -29: comparison with type I interferons. Cytokine 31, 109-118 (2005).

21. Khaitov, M.R. etal. Respiratory virus induction of alpha-, beta- and lambdainterferons in bronchial epithelial cells and peripheral blood mononuclear cells. Allergy 64, 375-386 (2009).

22. Bochkov, Y.A. et al. Rhinovirus-induced modulation of gene expression in bronchial epithelial cells from subjects with asthma. Mucosal Immunol. $\mathbf{3}$, 69-80 (2010).

23. Proud, D. et al. Gene expression profiles during in vivo human rhinovirus infection: insights into the host response. Am. J. Respir. Crit. Care Med. 178, 962-968 (2008).

24. Schoggins, J.W. et al. A diverse range of gene products are effectors of the type I interferon antiviral response. Nature 472 , 481-485 (2011)

25. Theofilopoulos, A.N., Baccala, R., Beutler, B. \& Kono, D.H. Type I interferons (alpha/beta) in immunity and autoimmunity. Annu. Rev. Immunol. 23, 307-336 (2005).

26. Lopez-Souza, N. et al. In vitro susceptibility to rhinovirus infection is greater for bronchial than for nasal airway epithelial cells in human subjects. J. Allergy Clin. Immunol. 123, 1384-1390 e1382 (2009).

27. Sykes, A. et al. Rhinovirus 16-induced IFN-alpha and IFN-beta are deficient in bronchoalveolar lavage cells in asthmatic patients. J. Allergy Clin. Immunol. 129, 1506-1514 e1506 (2012).

28. Gehlhar, K., Bilitewski, C., Reinitz-Rademacher, K., Rohde, G. \& Bufe, A. Impaired virus-induced interferon-alpha2 release in adult asthmatic patients. Clin Exp Allergy 36, 331-337 (2006).

29. Bufe, A., Gehlhar, K., Grage-Griebenow, E. \& Ernst, M. Atopic phenotype in children is associated with decreased virus-induced interferon-alpha release. Int. Arch Allergy Immunol. 127, 82-88 (2002).

30. Gill, M.A. et al. Counterregulation between the FcepsilonRI pathway and antiviral responses in human plasmacytoid dendritic cells. J. Immunol. 184, 5999-6006 (2010).

31. Cakebread, J.A. et al. Exogenous IFN-beta has antiviral and antiinflammatory properties in primary bronchial epithelial cells from asthmatic subjects exposed to rhinovirus. J. Allergy Clin. Immunol. 127, 1148-1154 e1149 (2011).
32. McNamara, P.S., Kicic, A., Sutanto, E.N., Stevens, P.T. \& Stick, S.M. Comparison of techniques for obtaining lower airway epithelial cells from children. Eur. Respir. J. 32, 763-768 (2008).

33. Fonceca, A.M., Flanagan, B.F., Trinick, R., Smyth, R.L. \& McNamara, P.S. Primary airway epithelial cultures from children are highly permissive to respiratory syncytial virus infection. Thorax 67, 42-48 (2012).

34. Lopez-Guisa, J.M. et al. Airway epithelial cells from asthmatic children differentially express proremodeling factors. J. Allergy Clin. Immunol. 129, 990-997.e6 (2012).

35. Vareille, M. et al. Impaired type I and type III interferon induction and rhinovirus control in human cystic fibrosis airway epithelial cells. Thorax 67 , 517-525 (2012).

36. Pizzichini, M.M. et al. Asthma and natural colds. Inflammatory indices in induced sputum: a feasibility study. Am. J. Respir. Crit. Care. Med 158, 1178-1184 (1998).

37. Hewson, C.A. et al. Rhinovirus induces MUC5AC in a human infection model and in vitro via NF-kappaB and EGFR pathways. Eur. Respir. J. 36, 1425-1435 (2010).

38. Laza-Stanca, V. et al. The role of IL-15 deficiency in the pathogenesis of virus-induced asthma exacerbations. PLoS Pathog. 7, e1002114 (2011).

39. Koltsida, O. et al. IL-28A (IFN-lambda2) modulates lung DC function to promote Th1 immune skewing and suppress allergic airway disease. EMBO Mol. Med. 3, 348-361 (2011).

40. Wang, Q. et al. MDA5 and TLR3 initiate pro-inflammatory signaling pathways leading to rhinovirus-induced airways inflammation and hyperresponsiveness. PLoS Pathog. 7, e10020702011).

41. Moffatt, M.F. et al. A large-scale, consortium-based genomewide association study of asthma. N. Engl. J. Med. 363, 1211-1221 (2010).

42. Moffatt, M.F. et al. Genetic variants regulating ORMDL3 expression contribute to the risk of childhood asthma. Nature 448, 470-473 (2007).

43. Gupta, A. et al. Relationship between serum vitamin D, disease severity, and airway remodeling in children with asthma. Am. J. Respir. Crit. Care Med. 184, 1342-1349 (2011).

44. Bush, A. \& Saglani, S. Management of severe asthma in children. Lancet 376, 814-825 (2010).

45. Bracken, M. et al. The importance of nurse-led home visits in the assessment of children with problematic asthma. Arch Dis. Child 94, 780-784 (2009).

46. Gielen, V., Johnston, S.L. \& Edwards, M.R. Azithromycin induces anti-viral responses in bronchial epithelial cells. Eur. Respir. J. 36, 646-654 (2010).

47. Johnston, S.L. \& Tyrrell, D.A. Diagnstic Procedures for Viral, Rickettsial and Chylamydial Infections. American Public Health Association, Washington, D.C., 2005)

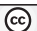

This work is licensed under the Creative Commons Attribution-NonCommercial-No Derivative Works 3.0 Unported License. To view a copy of this license, visit http:// creativecommons.org/licenses/by-nc-nd/3.0/ 